\title{
AVALIAÇÃO DO NÍVEL DE CONHECIMENTO DOS CONSUMIDORES DE UM SUPERMERCADO DA GRANDE SÃO PAULO SOBRE GORDURA TRANS
}

\section{EVALUATION OF THE LEVEL OF KNOWLEDGE OF THE CONSUMERS AT A SUPERMARIET AT THE GREAT SÃO PAULO AREA ON TRANS FAT}

\author{
Jéssica Dantas da Costa ${ }^{1}$; Maria Cristina de Almeida Gaspar ${ }^{1}$; Valdirene F. Neves \\ dos Santos ${ }^{1^{*}}$ \\ ${ }^{1}$ Universidade Paulista, Instituto de Ciências da Saúde, São Paulo, Brasil \\ *Autor correspondente: Valdirene Francisca Neves dos Santos . Telefone:011-98596-8855. \\ Universidade Paulista - Instituto de Ciências da Saúde - Rua Antonio Macedo, 505 - CEP \\ 03087 - 010 Parque São Jorge - SP - Brasil. e-mail: <val_usp@hotmail.com>
}

\begin{abstract}
RESUMO
Observa-se na atualidade uma modificação no hábito alimentar da população brasileira, com maior consumo de alimentos industrializados, muitas vezes com alta densidade energética, pobres em fibras, com elevado teor de sódio e de gorduras totais, além da presença de gordura trans.

É comprovado que o consumo de ácidos graxos trans (AGTs), de maneira intensa e em longo prazo, acarreta prejuízos à saúde, tais como aumento das frações de colesterol LDL (lipoproteína de baixa densidade) e diminuição do HDL (lipoproteína de alta densidade). O objetivo do estudo foi avaliar o nível de conhecimento dos consumidores de um supermercado da Grande São Paulo sobre gordura trans. Foi constatado desconhecimento acentuado sobre o ingrediente, suas fontes alimentares e seus malefícios à saúde, bem como a incapacidade de identificação de quais alimentos possuem a gordura trans em sua composição. Isso demonstra a necessidade de modificações quanto à padronização das recomendações e à forma de apresentação do termo "gordura trans" nas embalagens dos produtos expostos à venda.
\end{abstract}

Palavras-chave: Ácidos graxos trans; comportamento alimentar, alimentos industrializados.

\begin{abstract}
There is now a change in Brazilian eating habits, with higher consumption of industrialized foods, often high in calories, low in fiber, high in sodium and total fat and with the presence of trans fat.

It has been proven that the consumption of trans fatty acids (TFAs) in an intense way in the long term leads to health damage, such as an increase in LDL (low-density lipoprotein) cholesterol fractions and a decrease in HDL (high-density lipoprotein). This study aimed to evaluate the level of knowledge on trans fat among consumers in a supermarket at the Great São Paulo area. There was a marked lack of knowledge about the concept, its food sources, its health effects and the identification of which food products have trans fat in their composition. This demonstrates the need for modifications in the standards on recommendations, as well as the presentation of the term 'trans fat' on labeling of products on sale.
\end{abstract}

Key words: Trans fatty acids; food behavior; processed foods. 


\section{INTRODUÇÃO}

Observa-se na atualidade uma expressiva modificação no hábito alimentar da população. Fatores como distância entre o ambiente de trabalho e a residência e aumento do grupo feminino no mercado de trabalho foram determinantes para favorecer o aumento de restaurantes comerciais, institucionais e redes de fast food, sendo que muitas vezes as grandes refeições são substituídas por lanches (DAVID; GUIVANI, 2012).

Segundo a Organização Mundial da Saúde (WHO, 2001), um estilo de vida sedentário e uma dieta nutricionalmente desequilibrada são fatores associados às doenças cardiovasculares (DCV) e ao aumento de comorbidades. Isso ocorre devido à prática do consumo de alimentos com alta densidade energética, valores inadequados de fibras e teores elevados de sódio, carboidratos simples e gorduras saturadas, além da presença da gordura vegetal hidrogenada, também denominada gordura trans, que teve seu consumo aumentado a partir da década de 1950 em substituição à gordura saturada (DAVID; GUIVANI, 2012; MARTIN et al., 2004).

Os ácidos graxos trans (AGTs) não são sintetizados pelo organismo humano, mas podem ser obtidos a partir da bio-hidrogenação que ocorre no rúmen de animais. Portanto, quando um produto de origem animal é consumido, ocorre a ingestão de AGT (HARPER, 1994). Aproximadamente 90\% dos AGTs da dieta humana são provenientes de alimentos industrializados, o que demonstra a necessidade de maiores esclarecimentos por parte dos consumidores quanto à real quantidade desse composto presente nos produtos adquiridos em redes de supermercados (DAVID; GUIVANI, 2012. GAZZOLA; DEPIN, 2015).

A Resolução da Diretoria Colegiada n. 360 da Agência Nacional de Vigilância Sanitária (Anvisa) (BRASIL, 2003) estabeleceu que todos os alimentos embalados, fabricados a partir de 31 de julho de 2006, deveriam apresentar no rótulo a quantidade de gordura trans por porção e que, quando essa quantidade se apresentasse igual ou inferior a $0,2 \mathrm{~g}$ em $100 \mathrm{~g}$ ou 100 $\mathrm{ml}$, o produto poderia ser classificado como "isento" ou "livre".

Posteriormente, a Resolução da Anvisa n. 24 (BRASIL, 2010) dispôs que o alimento com quantidade elevada de gordura trans é aquele que possui em sua composição uma quantidade igual ou superior a
0,6 g de gordura trans por $100 \mathrm{~g}$ ou $100 \mathrm{ml}$ na forma como exposto à venda.

A presença do AGTs na dieta humana é um fator preocupante, uma vez que possui forte relação com o aumento do risco cardiovascular, elevando as frações de LDL (lipoproteína de baixa densidade) e diminuindo as frações de HDL (lipoproteína de alta densidade) (GAZZOLA; DEPIN, 2015).

Um estudo realizado por Santos (2015), que avaliou o grau de conhecimento dos consumidores sobre o que é gordura trans, mostrou que $41 \%$ dos entrevistados não sabiam o que é gordura trans.

Por meio da análise do conhecimento dos consumidores, é possível avaliar o nível de discernimento sobre os produtos habitualmente consumidos. Contudo, a falta de entendimento em relação aos componentes dos produtos adquiridos, aliada à dificuldade de interpretação do rótulo alimentício, frequentemente leva o consumidor a escolhas equivocadas e o mantém desinformado sobre as reais características dos alimentos consumidos.

Devido à relevância do assunto, o presente trabalho teve como objetivo avaliar o nível de conhecimento dos consumidores de um supermercado da Grande São Paulo sobre gordura trans.

\section{METODOLOGIA}

Trata-se de um estudo transversal de natureza descritiva, realizado em um supermercado na cidade de São Paulo. A pesquisa foi realizada a partir de aplicação de questionário, contendo questões objetivas, a fim de se avaliar o nível de conhecimento do consumidor sobre o tema abordado.

Na presente pesquisa, foram avaliadas as variáveis sexo, idade e grau de escolaridade, sendo os participantes da pesquisa indivíduos de ambos os sexos, com idade superior a 18 anos e alfabetizados. Após a aplicação do questionário, foi distribuído um folder com informações importantes sobre o tema para melhor compreensão dos participantes sobre o assunto.

A coleta de dados foi realizada em dias aleatórios da semana, nos períodos matutino e vespertino, sendo entrevistados 60 frequentadores de um supermercado na Grande São Paulo.

A tabulação de dados foi realizada no programa Microsoft Excel 2013, e os resultados foram apresentados na forma de gráficos para melhor visualização. 
O presente trabalho foi aprovado pelo Comitê de Ética em Pesquisa da Universidade Paulista (UNIP), sob o parecer número 1.915.239.

\section{RESULTADOS E DISCUSSÃO}

Os resultados obtidos foram classificados nos gráficos e tabelas abaixo. Na Tabela 1, pode-se verificar a caracterização sociodemográfica dos participantes. Observa-se que a maioria do grupo feminino entrevistado está na faixa etária de 31 a 40 anos $(40 \%)$ e que, do grupo masculino, de 18 a 30 anos (47\%).

Tabela 1. Caracterização Sociodemográfica dos Consumidores. São Paulo, 2017

\begin{tabular}{lcccc}
\hline Variável & \multicolumn{2}{c}{ n } & \multicolumn{2}{c}{$\%$} \\
\hline Sexo & $\mathrm{F}$ & $\mathrm{M}$ & $\mathrm{F}$ & $\mathrm{M}$ \\
Idade & $(30)$ & $(30)$ & $(50)$ & $(50)$ \\
18 a 30 & & & & \\
31 a 40 & 7 & 1 & 23 & 47 \\
41 a 50 & 12 & 9 & 40 & 30 \\
51 a 60 & 6 & 4 & 20 & 13 \\
61 a 70 & 3 & 3 & 10 & 10 \\
Grau de escolaridade & 2 & 0 & 7 & 0 \\
Ensino fundamental incompleto & & & & \\
Ensino fundamental completo & 3 & 1 & 10 & 3 \\
Ensino médio incompleto & 1 & 0 & 3 & 0 \\
Ensino médio completo & 5 & 3 & 17 & 10 \\
Ensino superior incompleto & 15 & 24 & 50 & 80 \\
Ensino superior completo & 2 & 2 & 7 & 7 \\
& 4 & 0 & 13 & 0 \\
\hline
\end{tabular}

F: Feminino

M: Masculino

Em relação ao grau de escolaridade, observaram-se, em ambos os grupos, maiores índices de participantes com ensino médio completo. O Gráfico 1 mostra que o número de homens (87\%) que anteriormente já tinham ouvido falar sobre gordura trans foi maior do que o de mulheres (73\%).
Gráfico 1. Avaliação percentual de consumidores, de acordo com o gênero, que já ouviram falar sobre gordura trans. São Paulo, 2017.

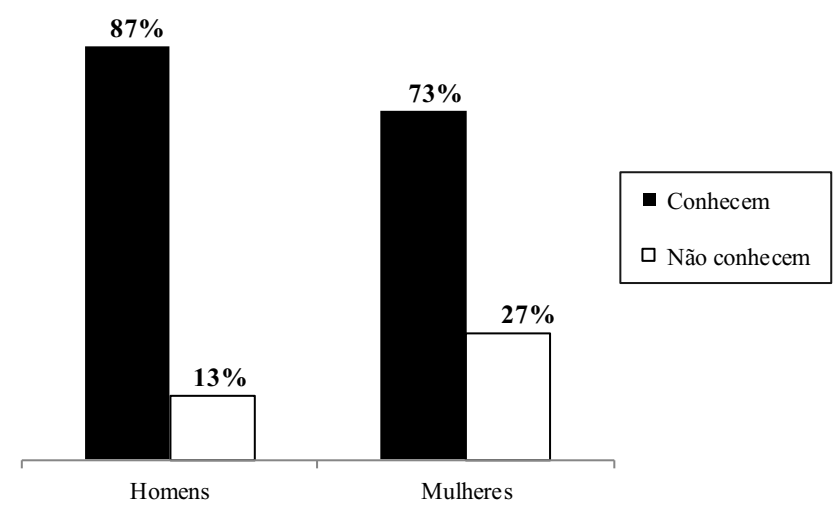

No Gráfico 2, vemos que a maioria dos consumidores não demonstrou conhecimento sobre o conceito de gordura trans, sendo que $90 \%$ dos homens e $97 \%$ das mulheres afirmaram não saber o que significa gordura trans. As respostas foram classificadas como negativas quando o entrevistado respondia "não" diretamente ou quando respondia incorretamente.

Gráfico 2. Avaliação percentual de consumidores de um supermercado, de acordo com o gênero, que conhecem ou não o conceito de gordura trans. São Paulo, 2017.

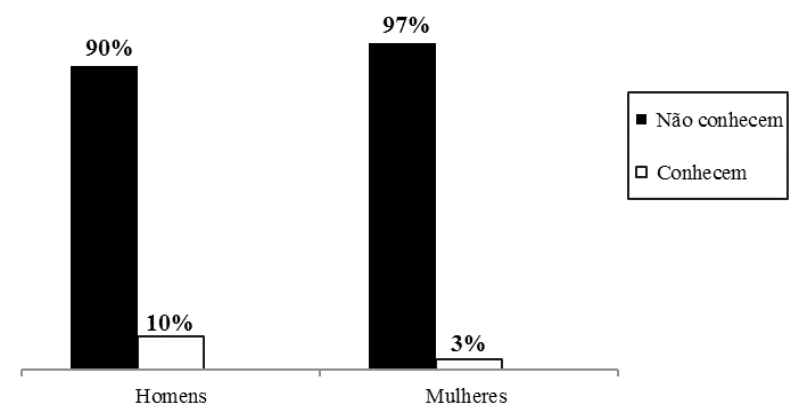

Estudo realizado por HEPP et al. (2007) que avaliou o conhecimento dos consumidores do município de Lajeado (RS) sobre gordura trans mostrou que apenas $14 \%$ dos consumidores entrevistados disseram ter conhecimento sobre o que são gorduras trans, sendo que, destes, $45 \%$ nunca ouviram falar sobre o tema. Ainda no mesmo estudo, a maioria dos consumidores (80\%) declarou não saber se os produtos comumente consumidos possuem ou não gordura trans em sua composição; apenas $1 \%$ referiu ter conhecimento dos produtos que possuem gordura trans, mas, devido ao sabor, afirmou manter o consumo (HEPP et al, 2007). 
A presente pesquisa, conforme se observa no Gráfico 3, 87\% das mulheres entrevistadas não sabem quais são os alimentos ricos em gordura trans, e esse percentual de desconhecimento foi ainda maior entre os homens (97\%).

Gráfico 3. Avaliação percentual do conhecimento de consumidores de um supermercado, de acordo com o gênero, sobre alimentos ricos em gordura trans. São Paulo, 2017.

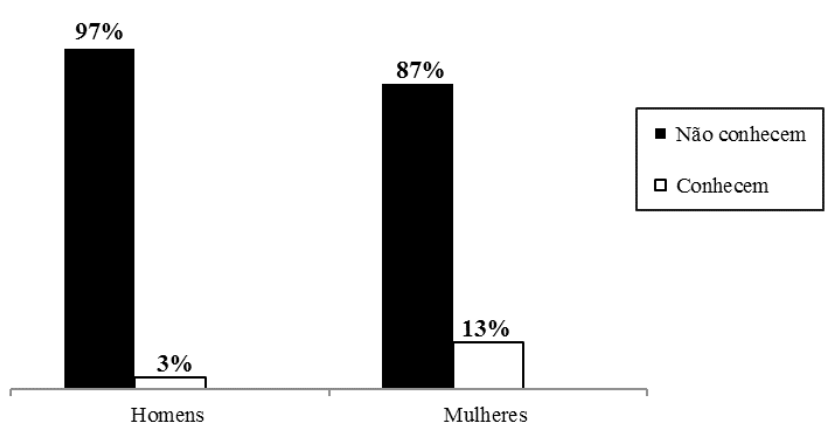

'Sabe-se que os alimentos que possuem maior quantidade de gordura trans em sua composição são os industrializados, também denominados de ultraprocessados. De acordo com o "Guia Alimentar para a População Brasileira”, alimentos ultraprocessados são formulados industrialmente e constituídos de substâncias extraídas de alimentos (óleos, gorduras, açúcar, amido, proteínas), derivadas de constituintes de alimentos (gorduras hidrogenadas e amido modificado) ou elaborados em laboratório (corantes, aromatizantes e realçadores de sabor) (MINISTÉRIO DA SAÚDE, 2014).

Embora o ultraprocessamento diminua a pericibilidade dos alimentos e os tornem agradáveis ao paladar, na maioria das vezes ele ocasiona a perda de nutrientes, água e fibras, além de concentrarem açúcar, sódio e gorduras acima dos limites preestabelecidos pela Organização Mundial da Saúde. Alimentos como fast-food, refrigerantes, salgadinhos de pacote, bolachas recheadas, sorvete, macarrão instâtaneo, chocolate e produtos semiprontos e prontos para consumo são exemplos dos alimentos ultraprocessados mais consumidos (ASBRAN, 2012).

Ao analisar o Gráfico 4, verificou-se que $77 \%$ das mulheres e $57 \%$ dos homens disseram não conhecer quais eram os malefícios à saúde do consumo de alimentos ricos em gordura trans.
Gráfico 4. Avaliação percentual do conhecimento dos consumidores de um supermercado, de acordo com o gênero, sobre os malefícios à saúde do consumo de alimentos ricos em gordura trans. São Paulo, 2017.

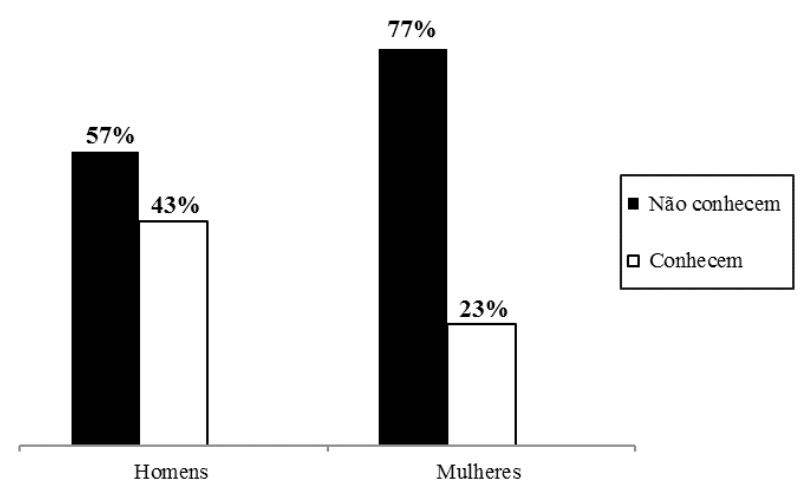

Pesquisa de Rocha e Partenez (2013) que avaliou o teor de AGTs em biscoitos e o consumo por frequentadores de um supermercado relacionou o maior grau de escolaridade com maior hábito de leitura dos rótulos alimentícios. A mesma relação também foi estabelecida em estudo de Hepp et al. (2007), que mostrou que a correlação entre o grau de escolaridade e o conhecimento sobre gordura trans é significativa, fato que demostra que quanto maior o grau de escolaridade, maior é o conhecimento sobre o tema abordado.

Sendo assim, os dados expostos no Gráfico 4 sugerem que os resultados deste estudo podem estar relacionados aos indivíduos entrevistados, ou seja, mulheres com ensino fundamental incompleto.

Atualmente, as doenças arteriais coronarianas são a principal causa de óbito no Brasil, sendo o aumento do colesterol o principal fator de risco modificável do tratamento não medicamentoso. $\mathrm{O}$ aumento dos níveis séricos de colesterol e de triglicerídeos são causados principalmente pelo maior consumo de alimentos com alto teor de ácidos graxos saturados, ácidos graxos trans e calorias (ARQUIVOS BRASILEIROS DE CARDIOLOGIA, 2013 ${ }^{\mathrm{a}}$.

Além de se relacionar com o aumento do colesterol total e do LDL e com a diminuição do HDL, o consumo de gordura trans está ligado ao aumento da resistência à insulina, podendo acarretar o desenvolvimento ou a progressão do diabetes mellitus tipo 2, bem como a elevação do risco de inflamação e o consequente aumento do risco cardiovascular(ARQUIVOS BRASILEIROS DE CARDIOLOGIA, 2013 ${ }^{\mathrm{b}}$. 
Nesta pesquisa, foi possível observar que $70 \%$ dos entrevistados não sabem como identificar se um alimento possui gordura trans em sua composição. A forma mais prática e segura de verificar a composição de um alimento industrializado é pela consulta à sua lista de ingredientes, sendo que os alimentos ultraprocessados tendem a possuir uma maior quantidade de ingredientes (cinco ou mais) (MINISTÉRIO DA SAÚDE, 2014).

Kraemer et al. (2011) cita que, para que haja uma leitura segura e confiável do rótulo alimentício, é necessário, além de consultar a informação nutricional, efetuar a leitura da lista de ingredientes - uma vez que a informação nutricional é apresentada por porção, que se refere à quantidade média do alimento que deve ser usualmente consumida por indivíduos saudáveis.

De acordo com o "Manual de Orientação aos Consumidores" da Agência Nacional de Vigilância Sanitária (Anvisa), o nutriente gordura trans não possui valor diário estabelecido, pois seu consumo não é recomendado (MINISTÉRIO DA SAÚDE, 2008).

\section{CONCLUSÃO}

Os dados obtidos na presente pesquisa indicam que os consumidores possuem um conhecimento deficiente sobre o conceito de gordura trans, suas fontes alimentares e seus malefícios à saúde. Além disso, uma quantidade expressiva de consumidores não consegue identificar se um alimento possui gordura trans em sua composição.

Concluímos que a divulgação da presença de gordura trans nos rótulos dos alimentos, inclusive em letras maiores nas embalagens e próximo à data de validade, deve ser feita de forma mais clara e precisa. Os autores sugerem alterações na legislação, de forma que o consumidor seja informado sobre a real quantidade de gordura trans que está sendo consumida (mesmo quando a quantidade de gordura trans na porção do alimento for inferior a $0,2 \mathrm{~g} / 100 \mathrm{~g}$ ).

\section{REFERÊNCIAS}

ARENHART, M. et al. A realidade das gorduras trans: conhecimento ou desconhecimento. Revista Disciplinarium Scientia. Série Ciências da Saúde, Santa Maria, v.10, n.1, p.59-63, 2009.

ASSOCIAÇÃO BRASILEIRA DE NUTRIÇÃO ASBRAN. Comida ultraprocessada e a epidemia de obesidade, 10 setembro 2012. Disponível em: <http:// www.asbran.org.br/noticias.php?dsid=887> Acesso em 09 abr. 2017.

BRASIL. Agência Nacional de Vigilância Sanitária (ANVISA). Resolução da Diretoria Colegiada $n^{\circ} 24$, Dispõe sobre a oferta, propaganda, publicidade, informação e outras práticas correlatas cujo objetivo seja a divulgação e a promoção comercial de alimentos considerados com quantidades elevadas de açúcar, de gordura saturada, de gordura trans, de sódio, e de bebidas com baixo teor nutricional. Diário oficial da União, 15 jun. 2010. Disponível em: < http://portal.anvisa. gov.br> Acesso em 20 nov. 2016.

BRASIL. Agência Nacional de Vigilância Sanitária (ANVISA). Resolução da Diretoria Colegiada $n^{0} 360$, Regulamento Técnico sobre Rotulagem Nutricional de Alimentos Embalados, tornando obrigatória a rotulagem nutricional. Diário Oficial da União, $23 \mathrm{dez}$. 2003. Disponível em: $<$ http://portal.anvisa.gov.br $>$ Acesso em 13 out. 2016.

BRASIL. MINISTÉRIO DA SAÚDE. Secretaria de Atenção à Saúde. Departamento de Atenção Básica. Guia alimentar para a população brasileira. 2. ed., p. 1-156, 2014.

DAVID M, GUIVANI J. A gordura trans: entre as controvérsias científicas e as estratégias da indústria alimentar. Revista Política e Sociedade, Florianópolis, v. 11, n.20, p. 214-224, 2012.

GAZZOLA, J.; DEPIN, H.M. Associação entre consumo de gordura trans e o desenvolvimento de doenças cardiovasculares (DCV). Revista Eletrônica de Extensão, Florianópolis, v. 12, n.20, p. 90-102, 2015.

HARPER, H.A. Manual de química fisiológica. São Paulo: Atheneu, 7. ed., 1994.

HEPP, K. et al. Avaliação do consumo de margarinas e o conhecimento sobre gordura trans dos consumidores do município de Lageado, RS. Revista Ciência, Biologia e Saúde, Londrina, v. 9, n.1, p.45-52, 2007.

KRAMER, M. et al. Relação entre a presença de gordura trans e o tamanho da porção em rótulos de produtos alimentícios. In: CONGRESSO NACIONAL DA SOCIEDADE BRASILEIRA DE ALIMENTAÇÃO E NUTRIÇÃO - SBAN, Fortaleza, v.11, p. 23-211, 2011.

MINISTÉRIO DA SAÚDE. Agência Nacional de Vigilância Sanitária (ANVISA): Manual de Orientação aos Consumidores. Universidade de Brasília - DF, p.1-24, 2008. Disponível em $<$ http://portal.anvisa.gov.br $>$. Acesso em 27 mar. 2017.

ROCHA, G.; PARTENEZ, A. Avaliação do teor de ácidos graxos trans em biscoitos e avaliação do consumo por frequentadores de um supermercado de São Paulo. Revista Simbio-logias, São Paulo, v. 6, n. 9, p. 43-656, 2013. 
SANTOS, J.S. Conhecimento sobre gordura trans em frequentadores de supermercados em Cariacica - ES. Trabalho de conclusão de curso, Faculdade Católica Salesiana, 2015.

SOCIEDADE BRASILEIRA DE CARDIOLOGIA (SBC). I Diretriz sobre o Consumo de Gorduras e Saúde Cardiovascular. Arquivos Brasileiros de Cardiologia, p.140, 2013.

SOCIEDADE BRASILEIRA DE CARDIOLOGIA (SBC). V Diretriz Brasileira de Dislipidemias e Prevenção da Aterosclerose. Arquivos Brasileiros de Cardiologia, p. $1-22,2013$.

WHO (World Health Organization). Manual on monitoring cardiovascular diseases. Washington: WHO, 2001. 